\title{
Quitter son emploi pour devenir entrepreneur
}

\author{
Inès Gabarret, EDC Paris, France \\ ines.gabarret@edcparis.edu \\ Benjamin Vedel, IAE Université Lille 1, France \\ benjamin.vedel@iae.univ-lille1.fr
}

\section{Résumé}

L’impulsion donnée par les gouvernements à la création d'entreprise, d'un côté, et les mutations de l'environnement de travail, de l'autre, affaiblissent la perception de valeur de la carrière traditionnelle. Dans ce contexte, la création d'entreprise se présente comme une alternative dans une trajectoire professionnelle. L'objectif de ce texte est de comprendre pourquoi certains cadres salariés décident de quitter leurs emplois pour créer leurs entreprises et oriente notre recherche sur l'analyse de la motivation entrepreneuriale. Nous verrons que la motivation entrepreneuriale des cadres hautement qualifiés est imparfaitement retranscrite par les théories existantes de la littérature en entrepreneuriat (push ou pull). Pour appréhender ce phénomène, 10 études de cas ont été menées. Les résultats montrent que la motivation entrepreneuriale du cadre salarié est composée de deux groupes de facteurs aux caractéristiques non économiques : l'insatisfaction dans leur relation au travail, et le désir d'indépendance. Il apparaît aussi que la motivation entrepreneuriale ne peut être expliquée suivant une logique de choix exclusif de variables mais correspond plutôt à une composition particulière propre à chaque individu. Sur ces résultats, nous proposons d'interpréter la motivation entrepreneuriale à la lumière de deux continuums : push/pull et économique/nonéconomique. 
Mots-clés : motivation entrepreneuriale, insatisfaction au travail, indépendance, turnover, entrepreneuriat

\begin{abstract}
The stimulus given by governments to new business creation and the transformation of the working environment weaken the perception of value of corporate jobs. In this context, entrepreneurship appears as an alternative to a traditional career. We studied the entrepreneurial motivation of corporate executives in order to understand why some of them decide to leave their jobs to create new companies. This is a qualitative research, based on 10 French managers who have left their jobs to become entrepreneurs. In this article we will see that the entrepreneurial motivation theory (push and pull) does not explain the non-economic aspects of the motivation of highly qualified managers.
\end{abstract}

The results of our study show that the entrepreneurial motivation could be described as two non-economical dimensions: dissatisfaction with the job, and desire of independence. Following this, we propose to remove the character of exclusivity in the choice of motivational factors, and to interpret the entrepreneurial motivation in the light of two continuums (push/pull and economic/non-economic).

Keywords: entrepreneurial motivation, dissatisfaction, independence, turnover, entrepreneurship

\title{
Introduction
}

Les impulsions données par les gouvernements, depuis plus de 30 ans, vers l'entrepreneuriat (principalement comme un remède anti-chômage) ont permis de placer la création d'entreprise comme une alternative de la carrière salariale. L'entrepreneuriat peut 
être compris comme un processus de création d'entreprise (ex-nihilo, essaimage, etc.) ou comme un rachat d'une société existante (reprise d'entreprise) et correspond à un mouvement et à un changement pour l'individu (Fayolle, 2005). Ce mouvement repose sur des motivations et arrive rarement par accident (Schjoedt \& Shaver, 2007). Il exige une volonté soutenue exercée au fil du temps.

Parallèlement à cela, les mutations de l'environnement de travail (telles que les restructurations, les fusions d'entreprises, et la nouvelle flexibilité de l'emploi, entre autres) ont affaibli la perception que les individus avaient de la carrière traditionnelle. Les représentations du travail sont ainsi devenues « de moins en moins uniformes et de plus en plus éclatées » (Thévenet, 2001, p.6).

Dans cet environnement organisationnel difficile, le «turnover» des employés reste, pour les entreprises, une des questions primordiales de la gestion des ressources humaines. Le turnover mesure les changements qui se produisent parmi les employés au cours d'une période donnée. Le départ de l'entreprise peut prendre son origine dans la volonté de l'individu, dans le cas d'une démission, ou ne pas être lié à cette volonté, dans le cas d'un licenciement (Bluedorn, 1978). Le turnover touche toutes les organisations et devient un problème majeur quand il s'agît des professionnels qualifiés (Besseyre des Horts \& Nguyen, 2010 ; Morin \& Renaud, 2009).

Lors du départ volontaire des salariés, plusieurs possibilités existent. Certains salariés se réorientent vers une autre relation d'emploi, tandis que d'autres se dirigent vers la création d'entreprise. Dans cet article, nous nous intéressons exclusivement au turnover volontaire des cadres salariés vers la création d'entreprise. En effet, d'après l'enquête réalisée par l'INSEE en $2002^{1}$, plus de $60 \%$ des créateurs et repreneurs en France étaient des salariés ou d'anciens

\footnotetext{
${ }^{1}$ Enquête SINE 2002 sur les nouvelles entreprises.
} 
salariés (parmi lesquels $18 \%$ de cadres et 22\% d'agents de maîtrise ou de professions intermédiaires).

Ce texte a pour objectif d'analyser la motivation entrepreneuriale d'un groupe atypique d'entrepreneurs (les cadres salariés qui décident de quitter leurs postes pour créer une entreprise), pour en souligner les caractéristiques et les particularités. Notre échantillon a été réduit à cette seule catégorie de personnes. Plusieurs entretiens biographiques ont été menés afin d'explorer les facteurs ou les situations qui conditionnent le départ volontaire du cadre salarié vers l'entrepreneuriat. Plus particulièrement, nous avons essayé de répondre aux questions suivantes :

$\checkmark$ Quelles sont les conditions qui vont favoriser l'émergence de la décision de démission du cadre d'entreprise ?

$\checkmark$ Quels sont les facteurs qui vont déclencher la motivation entrepreneuriale du cadre salarié ?

Les recherches précédentes sur la motivation entrepreneuriale considèrent que la création d'entreprise peut être choisie ou subie. Selon cette optique, elle peut être déclenchée par la présence d'une opportunité d'affaire, ou par un besoin de rémunération en cas de chômage. Ces deux postures exclusives ont donné naissance aux concepts économiques de la motivation entrepreneuriale «pull » et « push », ainsi qu'aux notions d'entrepreneur par opportunité et par nécessité.

Si beaucoup d'auteurs ont étudié la motivation à la création de l'entrepreneur traditionnel (Arenius \& Minniti, 2005 ; Carsrud \& Brännback, 2011 ; Hessels et al., 2008 ; Naffziger et al., 1994 ; Segal et al., 2005 ; Shane et al., 2003), peu ont visé le salarié devenu entrepreneur. Dans le cas spécifique de la motivation entrepreneuriale de l'employé qui quitte son poste pour créer une entreprise, deux visions s'affrontent. D'un côté, Brockhaus (1980), 
Cooper (1971) et Stoner \& Fry (1982), entre autres, indiquent que la motivation entrepreneuriale de l'employé repose sur une insatisfaction au travail. C'est cette insatisfaction qui va pousser l'individu vers la création d'entreprise. D'un autre côté, c'est la découverte d'une opportunité d'affaires qui va motiver le salarié vers l'entrepreneuriat (Shane \& Venkataraman, 2000).

D’autres auteurs commencent à questionner l'exclusivité des catégories push et pull, (Hughes, 2003 ; Kirkwood, 2009 ; Stevenson, 1990 ; Verheul et al., 2010) et considèrent que la motivation doit être vue comme un continuum le long duquel des combinaisons de facteurs de poussée et d'attraction existent.

Dans ce texte, nous verrons que la motivation entrepreneuriale des cadres hautement qualifiés est imparfaitement retranscrite par le découpage classique économique push/pull (que les catégories soient considérées comme exclusives ou non) et demande un éclairage nouveau. Nous proposons de l'interpréter à la lumière de deux continuums : push/pull et économique/non-économique. Nos résultats montrent que la motivation à la création de ces derniers se présente essentiellement sous la forme d'une composition de variables non économiques d'insatisfaction (push) et de désir d'indépendance (pull). Ce groupement de facteurs non exclusif fait référence au travail effectué précédemment en entreprise, aux objectifs, désirs et personnalité de l'individu, ainsi qu'à l'environnement hors de l'entreprise et permet des assemblages particuliers de facteurs propres à chaque individu.

Cet article s’organise en quatre parties. Après avoir précisé quelques éléments de définition en première partie, nous présentons en deuxième partie le choix de la méthode inductive et les dix cas d'étude. La troisième partie propose une analyse des facteurs repérés. Différentes pistes de recherche seront discutées dans la dernière partie.

\section{La motivation entrepreneuriale}


La recherche sur la motivation entrepreneuriale est divisée en deux corps théoriques principaux, nommés « push » et « pull » (Gilad \& Levine, 1986 ; Schjoedt \& Shaver, 2007). Ces théories donnent naissance à deux types de motivation : «par opportunité », dans le cas de la théorie pull, et «par nécessité », dans le cas de la théorie push (Acs et al., 2005 ; Reynolds et al., 2002). L'opportunité fait référence à la découverte d'une occasion d'affaires, alors que la nécessité correspond à une absence d'alternatives (McMullen et al., 2008).

La théorie «pull » postule que les opportunités d'affaires attirent les individus vers l'entrepreneuriat (Shane \& Venkataraman, 2000). Les facteurs d'attraction sont souvent regardés de manière plus positive car ils considèrent la création d'entreprise comme un choix individuel et volontaire (Kirkwood \& Campbell-Hunt, 2007). La reconnaissance d'opportunités est donc le trait caractéristique de l'entrepreneur (Shane \& Venkataraman, 2000) et est fortement corrélée à la décision de commencer une affaire (Arenius \& Minniti, 2005 ; Langowitz \& Minniti, 2007). Par ailleurs, dans la littérature, le terme opportunité a été largement utilisé (Casson \& Wadeson, 2007) et peut se définir comme une possibilité d'amélioration de revenus (Shane et al., 2003). Ainsi, l'entrepreneur opportuniste, comme décrit par Reynolds et al. (2002), est conduit par le désir et la recherche d'un gain économique (Carsrud \& Brännback, 2011).

Cependant, certains entrepreneurs peuvent être attirés vers la création d'entreprise par un désir d'indépendance, même si les gains économiques sont limités (Hughes, 2003). L'hypothèse d'une motivation non économique est aussi suggérée par Hessels et al. (2008). Dans leur étude, des entrepreneurs indépendants ne semblent pas avoir été motivés par la recherche d'une augmentation de richesse, mais plutôt par le fait d'avoir un niveau de vie confortable. 
D'autres recherches montrent que le besoin d'accomplissement, la possibilité de créer son propre emploi, et le désir d'autonomie se placent souvent au premier rang des citations de la motivation des créateurs (Verstraete \& Saporta, 2006). Ainsi, parmi les lifestyle entrepreneurs, et dans le cas de l'entrepreneuriat social ou familial, il existe habituellement une claire orientation non économique de la motivation. Finalement, on peut considérer qu'un certain nombre d'individus deviennent des entrepreneurs même si d'autres options attractives d'emploi existent (Carsrud \& Brannback, 2011).

D’un autre côté, la théorie «push » postule que les individus sont poussés vers l'entrepreneuriat par des situations conflictuelles, comme la perte de l'emploi, ou l'insatisfaction dans leur travail précédent (Bradley \& Roberts, 2005 ; Brockhaus, 1980 ; Cooper, 1971 ; Stoner \& Fry, 1982). Les facteurs de poussée viennent souvent accompagnés de connotations négatives (Kirkwood \& Campbell-Hunt, 2007). L’entrepreneuriat «par nécessité » est une possibilité qui se présente quand d'autres options de travail sont absentes ou insatisfaisantes (McMullen et al., 2008).

Néanmoins, l'insatisfaction avec le travail précédent pourrait mener l'individu vers la recherche d'un autre poste. La question qui se pose, alors, est de savoir pourquoi des cadres salariés créent de nouvelles sociétés au lieu de rechercher un autre emploi. Selon Brockhaus (1980), la décision de créer une nouvelle activité, au lieu de chercher un poste, est liée à l'intensité de l'insatisfaction. D'après l'auteur, il est possible que des entrepreneurs auraient été si peu satisfaits avec l'emploi précédent, qu'ils auraient considéré peu probable de trouver un emploi satisfaisant dans une autre organisation (Brockhaus, 1980). L'intensité de l'insatisfaction a aussi été étudiée par Stoner \& Fry (1982). Ils ont suggéré une relation entre l'intensité de l'insatisfaction dans l'emploi précédent et le type d'affaire ou d'industrie choisie pour le projet de création. Une forte insatisfaction semble mener les individus vers un changement d'industrie. 
Le Global Entrepreneurship Monitor (GEM), un programme qui décrit et analyse l'activité entrepreneuriale à travers le monde (Acs et al., 2005), respecte cette double typologie, push et pull, dans leurs études sur la motivation d'entreprendre. Comme ils le définissent, la motivation entrepreneuriale est basée sur la nécessité ou sur l'amélioration (économique) au travers d'une opportunité (Kelley et al., 2011). Cependant, comme ils le reconnaissent, le fait que cette typologie soit mutuellement exclusive pourrait mener à une mauvaise interprétation de la motivation dans certains cas. Ainsi, des individus ne se sentant représentés par aucune des deux postures, auraient une tendance à choisir des facteurs pull pour expliquer leur motivation entrepreneuriale (Bosma et al., 2009). Face à cette problématique, d'autres auteurs considèrent que la motivation doit être vue comme un continuum le long duquel des combinaisons de facteurs de poussée et d'attraction existent (Hughes, 2003 ; Kirkwood, 2009 ; Stevenson, 1990 ; Verheul et al., 2010).

La motivation entrepreneuriale est, donc, un sujet complexe (Kirkwood \& CambellHunt, 2007). Cependant, au-delà de cette complexité, certains éléments y apparaissent régulièrement (Kuratko et al., 1997) : (1) l'importance d'une récompense extrinsèque monétaire ; (2) le besoin d'une récompense intrinsèque liée à l'accomplissement d'une tâche ; (3) la recherche de liberté et d'indépendance ; et (4) la perte d'emploi. La littérature précise ainsi que les individus deviennent des entrepreneurs pour des raisons multiples, et que des motifs non-économiques rivalisent fortement avec les motifs économiques (Cromie, 1987).

\section{Méthode de recherche}

Cette étude, à visée compréhensive et interprétative, est fondée sur une méthode qualitative. Comme l'indique Kirkwood (2009), les méthodes qualitatives sont mieux adaptées pour capturer la complexité de la motivation entrepreneuriale. La recherche a été conduite suivant les principes de la théorie enracinée (Strauss \& Corbin, 2004) et a été basée 
sur une analyse approfondie à partir des histoires de vie de 10 cadres français, ayant démissionné pour créer leurs entreprises.

La méthodologie de récit de vie s'avère particulièrement convenable pour l'étude de l'entrepreneuriat (McKenzie, 2005). Elle est aussi utile quand il s'agît d'interpréter le sens donné par les individus à leurs carrières, aussi bien de manière générale que dans le cas spécifique d'une transition de carrière (Cohen \& Mallon, 2001).

Le récit de vie permet à l'individu de raconter son expérience. En le faisant, il établit un ordre d'événements non nécessairement chronologique. Par cette méthode, il peut aussi révéler des incohérences et contradictions liées à son expérience de travail. Le récit de vie implique un processus de fabrication de sens rétrospectif permettant de comprendre la relation que l'interviewé tisse avec les structures sociales (Cohen \& Mallon, 2001).

Notre collecte de données a été effectuée à partir d'entretiens biographiques (récit de vie) pour permettre aux individus de contextualiser et hiérarchiser les événements significatifs.

Lors du récit des interviewés, nous avons cherché à identifier certains aspects relatifs au changement. Une fois que l'individu avait fini de raconter son expérience, si ces sujets n’avaient pas été abordés de façon spontanée, des relances étaient proposées («Pouvez-vous aussi nous raconter ... »). Dans la plupart des cas les relances n’ont pas été nécessaires. Les thèmes attendus sont listés ci-dessous :

Faits/situations en rapport avec le plaisir/déplaisir dans la relation d'emploi ;

$\checkmark$ Objectifs et attentes au travail ;

Motivations de quitter l'entreprise ; 
$\checkmark$ Motivations de créer une entreprise.

Les entretiens se sont déroulés dans les locaux de travail des entrepreneurs. Ils ont duré entre une et deux heures, ont été enregistrés et retranscrits dans des documents Word. Une étude thématique des transcriptions a été achevée identifiant et codant le discours dans des unités de sens. Les données retranscrites et analysées ont été transférées vers le logiciel NVIVO International QSR 7 pour leur organisation et la présentation des résultats. Les unités de sens ont été étiquetées comme des nœuds. Nous avons, ensuite, identifié les relations.

L'analyse exploratoire a été effectuée sur un échantillon de 10 individus. Ne prétendant à aucune forme de représentativité statistique, les critères suivants ont été retenus pour la sélection des cas :

$\checkmark$ L'individu possède une expérience professionnelle de carrière reconnue, et avait un statut de cadre avant la création d'entreprise ;

$\checkmark$ L'individu a décidé de quitter son poste précédent sans avoir été licencié ;

$\checkmark$ L'individu a déjà créé son entreprise et il y travaille actuellement.

Tableau 1 : L'échantillon 


\begin{tabular}{|l|c|c|}
\hline Caractéristiques & $\begin{array}{c}\text { Hommes } \\
(\boldsymbol{n}=7)\end{array}$ & $\begin{array}{c}\text { Femmes } \\
(\boldsymbol{n}=\mathbf{3})\end{array}$ \\
\hline Agés entre 30 et 40 ans & 2 & 2 \\
\hline Agés entre 40 et 50 ans & 4 & 0 \\
\hline Agés de plus de 50 ans & 1 & 1 \\
\hline Expérience salariale en entreprise commerciale & 0 & 2 \\
\hline Expérience salariale en entreprise de services & 3 & 1 \\
\hline Expérience salariale en entreprise de technologie & 3 & 0 \\
\hline Expérience salariale en autres types d'entreprises & 1 & 0 \\
\hline Création d'entreprise commerciale & 2 & 1 \\
\hline Création d'entreprise de services & 3 & 2 \\
\hline Création d'entreprise technologique & 1 & 0 \\
\hline Création d'autres types d'entreprises & 1 & 0 \\
\hline Niveau académique inférieur et égal à Bac+5 & 6 & 1 \\
\hline Niveau académique supérieur à Bac+5 & 1 & 2 \\
\hline
\end{tabular}

Le groupe étudié est composé de 3 femmes (F1 à F3) et 7 hommes (M1 à M7) choisis en utilisant la méthode de réseau ou boule de neige. Cette méthode d'échantillonnage non aléatoire s'avère utile lorsqu'on recherche une meilleure compréhension à propos d'un phénomène ou qu'on essaye d'améliorer les connaissances d'une théorie particulière (Onwuegbuzie \& Leech, 2007). Dans le cas de notre étude, le phénomène étudié était la transition de l'emploi cadre vers l'entrepreneuriat. Pour cela, il était important d'interroger des individus qui partageaient des caractéristiques similaires (telles que l'emploi cadre et la haute qualification), et qui avaient vécus des expériences comparables (comme la transition vers l'entrepreneuriat).

Une caractéristique de la méthode d'échantillonnage a été la composition d'un terrain mixte. Plusieurs études se sont intéressées à l'influence du genre sur la motivation de créer une entreprise, pourtant les résultats restent partagés et demandent des recherches supplémentaires (Kirkwood, 2009). Il apparait, néanmoins, que les entrepreneurs, en 
considération de leur genre (homme ou femme), possèdent des motivations entrepreneuriales relativement similaires (Kirkwood, 2009).

La composition de l'échantillon (30\% d'entrepreneurs féminins) s'est avérée cohérente avec la littérature, ainsi qu'avec les enquêtes de création d'entreprise. Les études antérieures montrent que, même si l'entrepreneuriat féminin est en constante augmentation, il continue d'être inférieur à l'entrepreneuriat masculin (Grilo \& Thurik, 2004 ; Kirkwood, 2009 ; Langowitz \& Minniti, 2007 ; Moult \& Anderson, 2005). En effet, d'après les pourcentages de la création d'entreprise, les femmes représentent $29 \%$ de l'entrepreneuriat dans l'Union européenne (Humbert \& Drew, 2010), 26\% au Royaume-Uni et 38\% aux USA (Carter, 2000).

\section{Résultats}

L'analyse thématique des données sur la motivation entrepreneuriale du cadre salarié a permis l'identification de groupements particuliers d'une multiplicité de facteurs nonéconomiques et une nouvelle interprétation des facteurs d'insatisfaction et de désir d'indépendance. Ces derniers sont liés principalement à : (1) une problématique d'insatisfaction dans leur rapport au travail en entreprise, et (2) un désir d'indépendance qui, partant des objectifs et caractéristiques individuelles de l'employé, se voit renforcé par certains aspects de l'environnement. Les facteurs motivationnels sont illustrés dans le tableau 2. 
Tableau 2 : Les variables d'insatisfaction et de désir d'indépendance

\begin{tabular}{|c|c|}
\hline Facteurs d'insatisfaction & Facteurs de désir d'indépendance \\
\hline L'ennui au travail & La recherche d'autonomie \\
\hline Les problèmes avec la hiérarchie & La possibilité de choisir son lieu de travail \\
\hline Le manque de respect au travail & $\begin{array}{l}\text { La liberté d'organiser son travail par rapport à la vie } \\
\text { familiale }\end{array}$ \\
\hline Le favoritisme pour certains au détriment d'autres & La liberté de la création \\
\hline L'instabilité professionnelle & $\begin{array}{l}\text { La possibilité de choisir, voire réduire, la quantité de } \\
\text { temps au travail }\end{array}$ \\
\hline Le plafond de carrière & La liberté de faire ce dont on est capable \\
\hline La stagnation par l'âge & $\begin{array}{l}\text { Le choix de faire, et réussir, comme ceux qui ont } \\
\text { réussi }\end{array}$ \\
\hline
\end{tabular}

La motivation entrepreneuriale du cadre salarié se révèle comme une motivation non économique. En effet, en raison des caractéristiques spécifiques du terrain (cadres avec pouvoir, statuts, hauts revenus, etc.), l'augmentation du revenu n'est pas un facteur de motivation pour le changement de carrière. Dans la plupart des cas, il apparaît que, pour les cadres salariés, le passage vers l'entrepreneuriat ne permet pas d'obtenir un niveau de revenus supérieurs. [De toutes façons je n'ai pas besoin de revenus extrêmement importants et du coup, je n'ai pas besoin de 50 Rolex et 3 bateaux (M3)], [Moi, je connais très exactement la fourchette d'argent qu'il me faut pour vivre superbement, au-delà ce sont des embêtements (M7)]. Cette motivation non économique se voit représentée par une multiplicité de variables d'insatisfaction et de désir d'indépendance mentionnées lors des discours des interviewés.

\section{La diversité des facteurs d'insatisfaction}

Comme nous l'avons présenté dans le tableau 2, l'insatisfaction est composée de sept variables non économiques, dont l'ennui au travail, les problèmes avec la hiérarchie, le manque de respect, le favoritisme pour certains au détriment d'autres, l'instabilité professionnelle, le plafond de carrière, et la stagnation par l'âge. L'ennui au travail fait référence à un sentiment de lassitude, d'inutilité, ou à un manque d'intérêt pour la tâche, et se manifeste comme la variable d'insatisfaction la plus citée dans l'étude (5 cas sur 10, dont 
deux tiers de femmes). Ce concept n'a pas été beaucoup développé dans la littérature, apparaissant discrètement comme un antécédent de la création entrepreneuriale dans une étude de Bradley \& Roberts (2004) et dans une autre de Hisrich \& Brush (1985). [Je faisais mon travail, tout le monde était très satisfait, on n'avait pas de problèmes, mais moi je cherchais autre chose, je le sentais (...) je cherchais plus à prendre mon temps et à trouver quelque chose de plus épanouissant intellectuellement (F2)], [Je peux rester deux ou trois ans à faire la même chose mais après je commence à m'ennuyer et il faut que je change, je suis toujours en recherche de nouveauté (M2)].

Deux autres variables ont été repérées parmi 4 des 10 cas étudiés. Celles-ci sont les problèmes avec la hiérarchie et le plafond de carrière. Contrairement aux attentes, aucune femme ne s'est manifestée par rapport au plafond de carrière. Cela viendrait en contradiction avec la littérature sur le plafond de verre (l'empêchement des femmes cadres à accéder au pouvoir dans les organisations). Pourtant, 4 hommes ont trouvé que leurs possibilités de carrière devenaient limitées. [Au bout de deux ans, je dirais qu'il n'y avait pas vraiment de progression, je dirais que d'un point de vue travail, j'ai commencé à être un petit peu limité (M5)]. En ce qui concerne les problèmes avec la hiérarchie, Bradley \& Roberts (2004) distinguent cette variable comme un antécédent de la création entrepreneuriale. Dans le cas de notre étude, ce qui ressort des récits de vie collectés, ce n'est pas un rejet de l'autorité, mais plutôt une critique liée à la façon dont l'autorité est administrée. Un abus d'autorité est mal toléré par des professionnels qui devraient jouir d'un peu plus d'autonomie, mieux en rapport avec leur niveau hiérarchique. [Je n'aimais surtout pas la manière autoritaire déployée (M1)]. En même temps, ces cadres sont censés pouvoir exprimer leurs idées. Pourtant, le fait de le faire peut provoquer des problèmes, dû à l'impossibilité de la hiérarchie d'accepter les critiques. [J'ai toujours eu un peu de problème avec la hiérarchie, parce que j'ai un caractère assez franc, c'est-àdire quand je trouve les choses qui ne sont pas bien, ou qui sont mal organisées, je le dis ouvertement et clairement (M5)]. 
Deux variables (favoritisme et instabilité) sont mentionnées par 3 des interviewés parmi les 10. Deux hommes et une femme ont manifesté la primauté d'une forme de politique informelle, ou lobbying, au sein de l'entreprise, nécessaire pour progresser dans la carrière. [Grimper les échelons ça ne m'a pas intéressé parce qu'après c'est très politique, trop politique dans l'entreprise (M2)], [Je suis arrivé, et je n'ai pas envie d'aller plus loin, parce qu'aller plus loin ça veut dire qu'il y a de la politique (...) c'est du vrai boulot politique, du lobbying (M3)]. Aller plus loin ne semblerait pas dépendre des capacités ou de l'effort engagé, mais plutôt des alliances informelles et du favoritisme politique. Ceci provoque de la déception et de l'insatisfaction.

L’instabilité du travail cadre est notée surtout dans les histoires de délocalisation d'entreprises, [La société avait décidé de déménager, de (ville 1) à (ville 2), alors ils ont licencié tout le monde à (ville 1) et ils ont réembauché à (ville 2) parce qu'ils ne voulaient pas payer le déménagement (M2)], et de plans de licenciements vécus dans l'entreprise, même si le cadre en a été épargné, [Il y avait une grosse réduction, quand je suis entré il y avait 330 employés et ils en ont gardé 110 (...) je suis allé directement voir mon chef, et j'ai dit, moi j'arrête, c'est bon, et j'ai plus envie de rester ici (M5)]. Le sentiment d'instabilité est provoqué par la perception d'un danger ou d'une menace vis-à-vis de la sécurité de l'emploi (Cromie, 1987). Mais aussi, par la constatation de la flexibilité du travail (Bridges, 1995). Elle provoque la désimplication et le désengagement des salariés (Dubouloy \& Fabre, 2002).

Deux dernières variables de l'insatisfaction sont mentionnées par seulement 2 interviewés. Il s'agit du manque de respect et de la stagnation par l'âge. [La dernière année ça s'est mal passée (...) c'est quelque chose au quotidien sur des petites choses, mais c'est non-stop, on demande toujours plus et on dévalorise de plus en plus (F1)]. Cette femme s'est retrouvée face à une situation qu'on peut définir comme du harcèlement moral. Une conduite de dévalorisation constante, pendant une longue période, de la part de la hiérarchie, visant la démotivation et la démission du cadre. Un autre cas de manque de respect a été répertorié, 
sans arriver à un tel extrême. [Donc, envie d'être cohérent avec moi-même, envie d'être respecté parce que je trouve que ce n'était pas une histoire d'argent mais de respect (M7)].

Toutes ces variables d'insatisfaction vont jouer un rôle dans la décision du cadre de démissionner. Pourtant, le départ volontaire n'implique pas une création postérieure d'entreprise. Pour arriver à cela, il faut également être en présence d'autres facteurs de niveau individuel et environnemental.

\section{Les facteurs multiples du désir d'indépendance}

Les cadres se déclarent motivés vers la création d'entreprise par un désir d'indépendance. Cette dimension est composée de 7 variables dont la recherche d'autonomie, la possibilité de choisir son lieu de travail, la liberté d'organiser son travail par rapport à la vie familiale, la liberté de la création, la possibilité de réduire la quantité de temps au travail, la liberté de faire ce dont on est capable, et le choix de faire comme ceux qui ont réussi.

Les trois variables d'attraction les plus cités par les interviewés (5 cas sur 10) ont été l'autonomie, la conciliation du travail avec la vie familiale, et la possibilité de choisir son lieu de travail. Pendant que la recherche d'autonomie est, sans doute, une variable classique d'attraction de l'entrepreneuriat, [J'ai monté mon entreprise parce que j'avais besoin d'autonomie, alors j'ai privilégié l'autonomie par rapport à un bon revenu (M7)], le fait de pouvoir choisir son lieu de travail se place à un même niveau d'importance dans les réponses de nos interviewés, [J'ai toujours voulu rester sur la région, cela a toujours été ma priorité, (cela a) toujours été quelque chose de claire et nette dans ma tête (M5)]. La problématique de la conciliation vie au travail/vie familiale n'est pas nouvelle. Plusieurs études la mentionnent parmi les principaux facteurs de motivation féminine (par exemple : Brockhaus, 1980, Cromie, 1987). Notre étude n'échappe pas à cette réalité : la totalité des femmes l'ont mentionnée comme étant d'importance dans leur choix entrepreneurial, [J'ai eu un premier enfant et j'ai dû changer parce que ce n'était plus 
possible continuer à travailler comme ça (F3)]. Pourtant, cette problématique a aussi intéressé quelques hommes de notre échantillon ( 2 sur 7). [J'avais envie de me désengager, en plus j'ai eu un enfant, donc j'avais envie de prendre un peu de distance pour avoir plus de temps pour ma famille (M1)].

Nous avons appelé auto-confiance la possibilité de faire ce dont on s'estime capable. Cette variable fait référence à la reconnaissance des capacités personnelles (Bandura, 1995), mais aussi à la possibilité de les mettre en œuvre pour réussir (Arenius \& Minniti, 2005 ; Bradley \& Roberts, 2004). La confiance en ses capacités est une des caractéristiques principales de l'entrepreneur (Langowitz \& Minniti, 2007), et a été soulignée par 4 hommes parmi nos 10 interviewés. [Il y a un marché, je le connais, parce que je le côtoie depuis des années, je sais les réseaux qui peuvent me prescrire de la clientèle (M4)], [Se mettre à son compte c'est un gros risque, je l'ai fait parce que je voulais, je me sentais capable (M6)].

Trois dernières variables ont aussi été mentionnées, pourtant dans une moindre mesure. Il s'agît, premièrement, de la possibilité de réduire le temps de travail en étant entrepreneur, variable repérée par une femme, [Je ne voulais (...) pas passer ma vie à travailler non plus (F1)], et par un homme, [ldéalement, je n'ai pas envie de travailler toute ma vie (M3)]. Face à la quantité importante du temps de travail exigée par les entreprises auprès de leurs cadres salariés, l'entrepreneuriat semblerait fournir une alternative plus équilibrée. La présence de « role models » dans leur environnement, soit familier, soit professionnel, peut aussi inspirer la création (Arenius \& Minniti, 2005 ; Langowitz \& Minniti, 2007 ; Shapero \& Sokol, 1982), et agir comme une force d'attraction vers l'entrepreneuriat. [J'aidais les entrepreneurs à s'implanter sur tous ce qu'on appelle les zones sensibles (...) quand on accompagne plein de personnes à se mettre à son compte, ça donne des idées, et donc je me suis mis à mon compte (M4)]. Finalement, un interviewé s'est manifesté attiré par le côté créatif de l'entrepreneuriat. [La motivation c'est de créer quelque chose, d'essayer de voir si mes idées sont bonnes, et de valoriser mes idées (M3)]. 


\section{Une combinaison d'insatisfaction et d'indépendance : Les mosaïques motivationnelles}

De l'analyse des discours, nous pouvons conclure que, dans le cas de notre échantillon de cadres salariés, la seule attraction pour l'entrepreneuriat ne suffit pas à déclencher la création. Si l'individu ne se trouve pas en présence des facteurs d'insatisfaction dans son travail, la création entrepreneuriale restera sous la forme d'un projet futur, pas nécessairement accompli. De la même manière, si un cadre salarié se trouve face à des facteurs de poussée mais ne se sent pas attiré par l'entrepreneuriat, son départ volontaire le conduira vers un autre emploi.

Ainsi, la motivation entrepreneuriale de chaque individu pourrait être interprétée comme une construction originale composée aussi bien de facteurs d'insatisfaction que d'indépendance. Cette composition de facteurs sera regroupée sous la forme d'une mosaïque motivationnelle déterminant la capacité de l'individu à aller vers la création d'entreprise.

Il y a des cas où la motivation est plus marquée par des variables d'insatisfaction, d'autres cas par des variables d'indépendance. Pourtant, dans tous les cas étudiés, on note une présence conjointe des variables des deux groupes.

Tableau 3 : Les mosaïques motivationnelles des interviewés 


\begin{tabular}{|c|c|c|c|c|c|c|c|c|c|c|}
\hline & F1 & F2 & M1 & M2 & M3 & M4 & M5 & M6 & F3 & M7 \\
\hline Insatisfaction & & & & & & & & & & \\
\hline Ennui & & $\mathbf{X}$ & & $\mathbf{X}$ & & & & $\mathbf{X}$ & $\mathbf{X}$ & $\mathbf{X}$ \\
\hline Hiérarchie & & & $\mathbf{X}$ & & & & $\mathbf{X}$ & & $\mathbf{X}$ & $\mathbf{X}$ \\
\hline Irrespect & $\mathbf{X}$ & & & & & & & & & $\mathbf{X}$ \\
\hline Favoritisme & & & & $\mathbf{X}$ & $\mathbf{X}$ & & & & $\mathbf{X}$ & \\
\hline Instabilité & & & & $\mathbf{X}$ & & $\mathbf{X}$ & $\mathbf{X}$ & & & \\
\hline Plafond de carrière & & & $\mathbf{X}$ & $\mathbf{X}$ & & & $\mathbf{X}$ & $\mathbf{X}$ & & \\
\hline Stagnation par l'âge & & & & & & & & $\mathbf{X}$ & $\mathbf{X}$ & \\
\hline Indépendance & & & & & & & & & & \\
\hline Autonomie & $\mathbf{X}$ & & & & $\mathbf{X}$ & & & $\mathbf{X}$ & $\mathbf{X}$ & $\mathbf{X}$ \\
\hline Lieu de travail & $\mathbf{X}$ & & & $\mathbf{X}$ & $\mathbf{X}$ & $\mathbf{X}$ & $\mathbf{X}$ & & & \\
\hline Vie familiale & $\mathbf{X}$ & $\mathbf{X}$ & $\mathbf{X}$ & & & $\mathbf{X}$ & & & $\mathbf{X}$ & \\
\hline Création & & & & & $\mathbf{X}$ & & & & & \\
\hline Temps au travail & $\mathbf{X}$ & & & & $\mathbf{X}$ & & & & & \\
\hline Auto-confiance & & & & $\mathbf{X}$ & $\mathbf{X}$ & & & $\mathbf{X}$ & & $\mathbf{X}$ \\
\hline Role models & & & & & & $\mathbf{X}$ & $\mathbf{X}$ & & & \\
\hline
\end{tabular}

Les mosaïques motivationnelles reprennent l'idée déjà développée par plusieurs auteurs de l'existence d'un continuum push/pull de la motivation. Cependant, dans le cas de notre étude, le continuum motivationnel est composé uniquement par des facteurs d'insatisfaction et d'indépendance, c'est-à-dire, qu'il ne comporte pas de facteurs économiques tels que la nécessité ou la découverte d'une opportunité d'enrichissement. Il s'agît d'un continuum push/pull non économique.

A partir de ce résultat, nous allons discuter de la nécessité d'incorporer un deuxième axe d'analyse, en forme d'un continuum économique/non économique, afin de mieux représenter la motivation entrepreneuriale.

\section{Discussion}


La caractéristique la plus marquante de notre étude est l'absence de facteurs de motivation du type économique dans le discours de nos interviewés. Cette absence peut s'expliquer par l'orientation de l'étude. La recherche a été limitée volontairement au cas des cadres salariés. Un cadre salarié jouit déjà, par sa position, d'un statut important au sein de l'entreprise, en même temps qu'il bénéficie d'un salaire élevé. Dans sa situation, le statut et le niveau économique sont des valeurs acquises. Dès lors qu'ils sont acquis, il n'y a plus de motivation à les acquérir, dans la mesure où le cadre salarié peut les conserver ou ne pas trop les diminuer. Le niveau économique et le statut continuent d'être des facteurs d'importance, pourtant ils ne sont pas mentionnés comme des déterminants de la motivation entrepreneuriale.

Comme nous l'avons déjà montré dans la revue de la littérature, la motivation entrepreneuriale peut s'interpréter à travers quatre dimensions, lesquelles ont été regroupées en deux corps mutuellement exclusifs (Figure 1) :

$\checkmark$ Push : correspondant à la nécessité (Acs et al., 2005 ; Reynolds et al., 2002), et à l'insatisfaction (Bradley \& Roberts, 2005 ; Stoner \& Fry, 1982 ; Brockhaus, 1980 ; Cooper, 1971) ;

$\checkmark$ Pull : correspondant à la recherche d'opportunités (Acs et al., 2005 ; Reynolds et al., 2002), et au désir d'indépendance (Hughes, 2003 ; Hessels et al., 2008).

Figure 1 : Les catégories exclusives de la motivation entrepreneuriale

\begin{tabular}{|c|c|}
\hline Push & Pull \\
\hline Nécessité / Insatisfaction & Opportunité / Indépendance \\
\hline
\end{tabular}

Or, notre étude exploratoire nous montre un regroupement de deux dimensions (insatisfaction et indépendance), qui pourrait correspondre à l'idée d'un continuum de 
motivation push/pull (Hughes, 2003 ; Kirkwood, 2009 ; Stevenson, 1990 ; Verheul et al., 2010) partiel et non économique (Figure 2).

Figure 2 : La motivation du cadre salarié devenu entrepreneur

\begin{tabular}{|c|c|}
\hline Push & Pull \\
\hline Nécessité & Opportunité \\
\hline Insatisfaction & Indépendance \\
\hline
\end{tabular}

Face à la complexité du sujet de la motivation entrepreneuriale (Kirkwood \& CambellHunt, 2007), une autre manière de l'évaluer nous semblerait pertinente. Ainsi, différentes pistes de recherches peuvent être formulées, afin d'élargir la compréhension de la motivation entrepreneuriale. La première, serait de l'étudier directement à partir de ses quatre dimensions principales : (1) le besoin d'un moyen de subsistance ou la création de son propre emploi face à un manque de possibilités sur le marché du travail, (2) l'envie d'améliorer ses revenus ou la possibilité d'exploiter une affaire hautement rentable, (3) la recherche d'un travail satisfaisant, où l'individu puisse exprimer toutes ses capacités et réaliser ses rêves professionnelles, et (4) le désir d'indépendance et d'autonomie, de n'avoir à rendre des comptes qu'à soi-même.

Une deuxième piste de recherche, en vue d'enrichir l'analyse, porterait sur la lecture des quatre dimensions principales de la motivation à la lumière de deux continuums (Figure 3) : (1) push/pull, (insatisfaction et nécessité versus indépendance et opportunité), et (2) économique/non-économique (nécessité et opportunité versus insatisfaction et indépendance).

Figure 3 : Les quatre dimensions de la motivation entrepreneuriale 


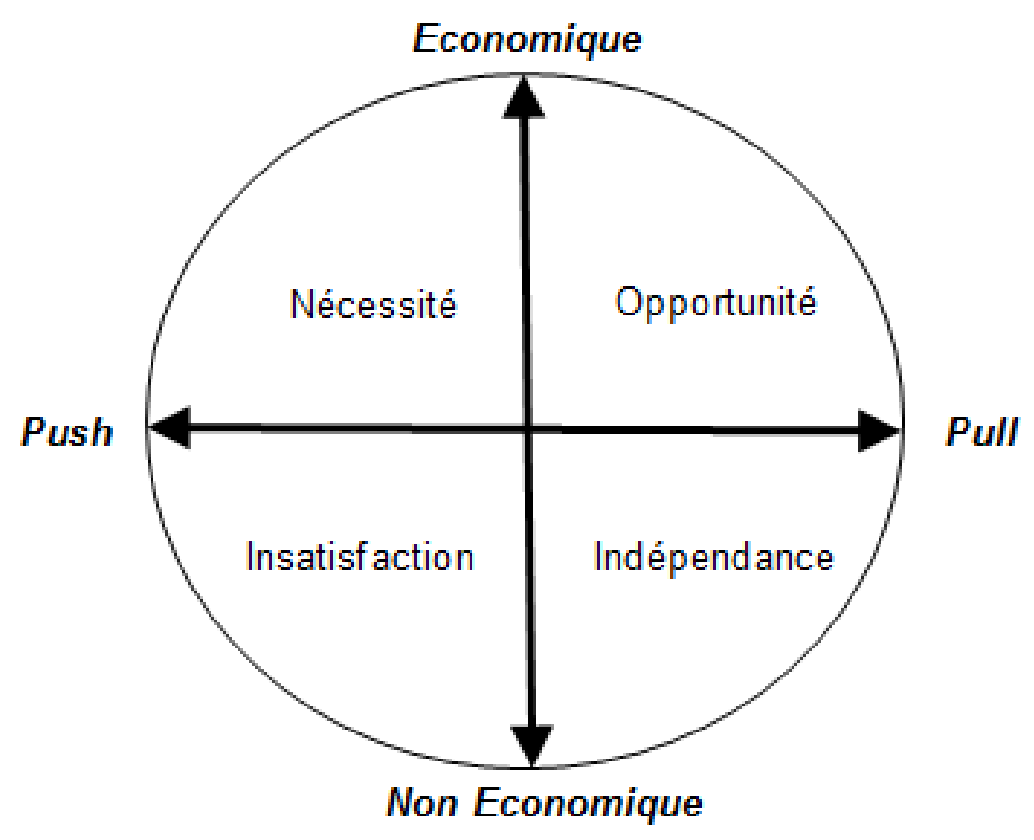

Nous avons souligné durant notre étude l'aspect personnel et multidimensionnel de la motivation entrepreneuriale. Un choix exclusif d'une des quatre dimensions au détriment des autres ne semble pas permettre d'expliquer correctement cette dernière. Il existe alors pour chaque individu une combinaison de facteurs particuliers qui le représente.

Ainsi, une troisième piste de recherche est avancée, celle de la non-exclusivité dans le choix de facteurs. Etant donné que les entrepreneurs sont un groupe d'individus de plus en plus hétérogène, la composition des facteurs motivationnels les représentant sera tout aussi hétérogène et dépendra, entre autres, des caractéristiques de personnalité de l'individu, de ses désirs et attentes, et des possibilités perçues dans l'environnement.

\section{Conclusion}

Cette recherche, qui a été menée de manière exploratoire sur un échantillon de 10 cadres moyens et supérieurs français ayant quitté leurs postes pour devenir entrepreneurs, propose une nouvelle manière de comprendre la motivation entrepreneuriale. 
Les résultats de l'étude montrent que la motivation entrepreneuriale du cadre salarié est composée d'une combinaison de deux dimensions non-économiques : l'insatisfaction dans leur relation au travail, et le désir d'indépendance. En effet, même si la question économique est importante, elle n'a pas constitué un facteur motivationnel pour le changement de carrière des managers de notre échantillon.

Deux contributions sont à retenir pour cette recherche. Du point de vue de l'accompagnement à la création d'entreprise, mieux comprendre la motivation de ce nouveau groupe d'entrepreneurs peut permettre aux collectivités de mieux les accompagner. Du côté des entreprises, une réflexion peut être ouverte sur la manière de conserver ses cadres et éviter la perte de talents, la réponse monétaire n'étant pas la plus appropriée. Ainsi, une partie de la motivation entrepreneuriale correspond à une érosion de la représentation que le cadre se fait de l'emploi salarié, et aboutit à son départ, en même temps que son intérêt pour la création grandit.

Finalement, cette décision peut être traduite comme un choix de carrière mais aussi un choix de vie, lié à une recherche d'autonomie et de satisfaction, et non pas forcement à un enrichissement économique. 


\section{Références}

Acs, Z., Arenius, P., Hay, M., \& Minniti, M. (2005). Global Entrepreneurship

Monitor, 2004 Executive Report. Babson College \& London Business School.

Arenius, P., \& Minniti, M. (2005). Perceptual Variables and Nascent Entrepreneurship. Small Business Economics, 24, 233-247.

Bandura, A. (1995). Self-Efficacy in Changing Societies. Cambridge University Press, UK.

Besseyre des Horts, C., \& Nguyen, V. (2010). Satisfaction, implication, engagement, enracinement et intention de départ des jeunes cadres : Une relation ambigüe. Congrès AGRH, 17-19 novembre, Rennes, Saint-Malo, France.

Bluedorn, A. (1978). A Taxonomy of Turnover. Academy of Management. The Academy of Management Review (pre-1986), 3, 000003, 647-651.

Bosma, N., Acs, Z., Autio, E., Coduras, A., \& Levie, J. (2009). Global Entrepreneurship Monitor, 2008 Executive Report. Babson College \& Universidad del Desarrollo.

Bradley, D., \& Roberts, J. (2004). Self-Employment and Job Satisfaction: Investigating the Role of Self-Efficacy, Depression, and Seniority. Journal of Small Business Management, 42, 1, 37.

Bradley, D., \& Roberts, J. (2005). Is Low Job Satisfaction a Cause of SelfEmployment Entrance? A Survival Analysis Using Data from the British Household Panel Survey. Working paper, East Carolina University, Greenville, North Carolina, 27 Mai.

Bridges, W. (1995). La conquête du travail, Au-delà des transitions. Ed. Village Mondial.

Brockhaus, R. (1980). The Effect of Job Dissatisfaction on the Decision to Start a Business. Journal of Small Business Management, 18, 37-43. 
Carsrud, A., \& Brannback, M. (2011). Entrepreneurial Motivations: What Do We Still Need to Know? Journal of Small Business Management, 49, 1, 9-26.

Carter, S. (2000). Improving the Numbers and Performance of Women-Owned

Businesses: Some Implications for Training and Advisory Services. Education \& Training, $42,4 / 5,326-333$.

Casson, M., \& Wadeson, N. (2007). The Discovery of Opportunities: Extending the Economic Theory of the Entrepreneur. Small Business Economics, 28, 285-300.

Cohen, L., \& Mallon, M. (2001). Using Stories as a Methodological Tool in Careers Research. International Studies of Management \& Organization, 31, 3, 48-68.

Cooper, A. (1971). The Founding of Technologically-Based Firms. The Center for Venture Management, Milwaukee, Wisconsin.

Cromie, S. (1987). Motivations of Aspiring Male and Female Entrepreneurs. Journal of Occupational Behavior, 8, 3, 251-261.

Dubouloy, M., \& Fabre, C. (2002). Les restructurations d'entreprises, de la rationalité économique à la souffrance des hommes. Gérer et Comprendre, 67, 43-55.

Fayolle, A. (2005). Introduction à l'entrepreneuriat. Dunod.

Gilad, B., \& Levine, P. (1986). A Behavioral Model of Entrepreneurial Supply. Journal of Small Business Management, October, 45-53.

Grilo, I., \& Thurik, R. (2004). Determinants of Entrepreneurship in Europe. Discussion Papers on Entrepreneurship, Growth and Public Policy, Max Planck Institute for Research into Economic Systems, Jena, July.

Hessels, J., Van Gelderen, M., \& Thurik, R. (2008). Entrepreneurial Aspirations, Motivations, and their Drivers. Small Business Economics, 31, 323-339.

Hisrich, R., \& Brush, C. (1985). Women and Minority Entrepreneurs: a Comparative Analysis. Frontiers of Entrepreneurship Research, 566-587. 
Hughes, K. (2003). Pushed or Pulled? Women's Entry into Self-Employment and Small Business Ownership. Gender, Work and Organization, 10, 4, 433-454.

Humbert, A., \& Drew, E. (2010). Gender, Entrepreneurship and Motivational Factors in an Irish Context. International Journal of Gender and Entrepreneurship, 2, 2, 173-196.

Kelley, D., Bosma, N., \& Amoros, J. (2011). Global Entrepreneurship Monitor: 2010. Global Report. Babson College, Universidad del Desarrollo \& London Business School.

Kirkwood, J. (2009). Motivational Factors in a Push-Pull Theory of Entrepreneurship. Gender in Management: An International Journal, 2, 5, 346-364.

Kirkwood, J., \& Campbell-Hunt, C. (2007). Using Multiple Paradigm Research Methodologies to Gain New Insights into Entrepreneurial Motivations. Journal of Enterprising Culture, 15, 3, 219-241.

Kuratko, D., Hornsby, J., \& Naffizger, D. (1997). An Examination of Owner's Goals in Sustaining Entrepreneurship. Journal of Small Business Management, January, 24-33.

Langowitz, N., \& Minniti, M. (2007). The Entrepreneurial Propensity of Women. Entrepreneurship Theory and Practice, May, 341-364.

McKenzie, B. (2005). Collecting Oral Histories for Entrepreneurship Research. New England Journal of Entrepreneurship, 8, 1, 37-48.

McMullen, J., Bagby, D., \& Palich, L. (2008). Economic Freedom and the Motivation to Engage in Entrepreneurial Action. Entrepreneurship Theory and Practice, September, 875895.

Morin, L., \& Renaud, S. (2009). La rétention des employés et les pratiques de formation et de rémunération : état des lieux de la littérature nord-américaine en GRH, psychologie et économie du travail. Congrès AGRH, 9-11 Septembre, Toulouse, France.

Moult, S., \& Anderson, A. (2005). Enterprising Women: Gender and Maturity in New Venture Creation and Development. Journal of Enterprising Culture, 13, 3, 255-271. 
Naffziger, D., Hornsby, J., \& Kuratko, D. (1994). A Proposed Research Model of Entrepreneurial Motivation. Entrepreneurship: Theory and Practice, 18, 3, $29-42$.

Onwuegbuzie, A., \& Leech, N. (2007). A Call for Qualitative Power Analyses. Quality \& Quantity, 41, 105-121.

Reynolds, P., Bygrave, W., Autio, E., Cox, L., \& Hay, M. (2002). Global Entrepreneurship Monitor, 2002 Executive Report. Babson College, Ewing Marion Kauffman Foundation, \& London Business School.

Segal, G., Borgia, D., \& Schoenfeld, J. (2005). The Motivation to Become an Entrepreneur. International Journal of Entrepreneurial Behaviour \& Research, 11, 1, 42-57. Schjoedt, L., \& Shaver, K. (2007). Deciding on an Entrepreneurial Career: A Test of the Pull and Push Hypotheses Using the Panel Study of Entrepreneurial Dynamics Data. Entrepreneurship Theory and Practice, September, 733-752.

Shane, S., \& Venkataraman S. (2000). The Promise of Entrepreneurship as a Field of Research. The Academy of Management Review, 25, 1, 217-226.

Shane, S., Locke, E., \& Collins, C. (2003). Entrepreneurial Motivation. Human Resource Management Review, 13, 257-279.

Shapero, A., \& Sokol, L. (1982). The Social Dimensions of Entrepreneurship. In Kent, C., Sexton, D., \& Vesper, K. (Ed.), Encyclopedia of Entrepreneurship, Prentice-Hall, Englewood Cliffs, New Jersey, 72-90.

Stevenson, L. (1990). Some Methodological Problems Associated with Researching Women Entrepreneurs. Journal of Business Ethics, 9, 439-446.

Stoner, C., \& Fry, F. (1982). The Entrepreneurial Decision: Dissatisfaction or Opportunity? Journal of Small Business Management, 20, 39-44. 
Strauss, A., \& Corbin, J. (2004). Les fondements de la recherche qualitative.

Techniques et procédures de développement de la théorie enracinée. Academic Press

Fribourg.

Thévenet, M. (2001). Le plaisir de travailler. Favoriser l'implication des personnes.

Editions d'Organisation.

Verheul, I., Thurik, R., Hessels, J., \& Van der Zwan, P. (2010). Factors Influencing the Entrepreneurial Engagement of Opportunity and Necessity Entrepreneurs. SCALES, Scientific Analysis of Entrepreneurship and SMEs, march.

Verstraete, T. \& Saporta, B. (2006). Création d'entreprise et entrepreneuriat. Editions de l'ADREG (http://adreg.net). 